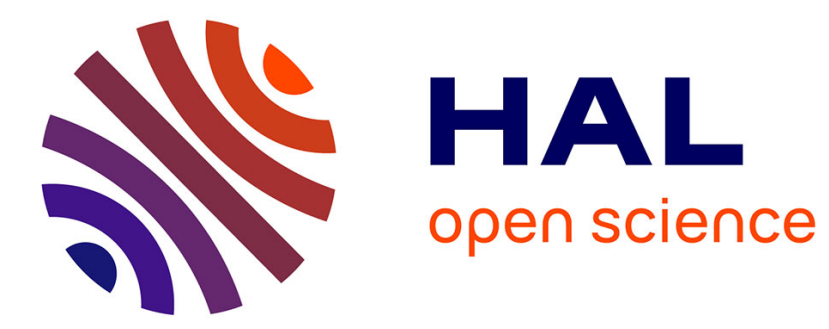

\title{
Nonlinear identification in structural dynamics based on Wiener series and Kautz filters
}

\author{
Samuel da Silva, Scott Cogan, Emmanuel Foltete
}

\section{To cite this version:}

Samuel da Silva, Scott Cogan, Emmanuel Foltete. Nonlinear identification in structural dynamics based on Wiener series and Kautz filters. Mechanical Systems and Signal Processing, 2010, 24 (1), pp.52-58. 10.1016/j.ymssp.2009.05.017 . hal-02300187

\section{HAL Id: hal-02300187 \\ https://hal.science/hal-02300187}

Submitted on 20 Nov 2019

HAL is a multi-disciplinary open access archive for the deposit and dissemination of scientific research documents, whether they are published or not. The documents may come from teaching and research institutions in France or abroad, or from public or private research centers.
L'archive ouverte pluridisciplinaire HAL, est destinée au dépôt et à la diffusion de documents scientifiques de niveau recherche, publiés ou non, émanant des établissements d'enseignement et de recherche français ou étrangers, des laboratoires publics ou privés. 


\title{
Nonlinear identification in structural dynamics based on Wiener series and Kautz filters
}

\author{
Samuel da Silva ${ }^{\mathrm{a}, *}$, Scott $\operatorname{Cogan}^{\mathrm{b}}$, Emmanuel Foltête ${ }^{\mathrm{b}}$ \\ a Western Paraná State University, UNIOESTE, Centro de Engenharias e Ciências Exatas, Av. Tancredo Neves, 6731, Parque Tecnológico Itaipu (PTI), \\ ZIP Code 85856-970, Foz do Iguaçu, Paraná, Brazil \\ b Institut FEMTO-ST, Département LMARC, Université de Franche-Comté - UMR 6174 24, rue de l'épitaphe, 25000 Besançon, France
}

The present paper proposes a method to identify localized nonlinear parameters in structural dynamics using vibration data. The approach is based on the identification of the first and second-order Volterra kernels in an orthogonal basis, namely Wiener kernels, while taking the experimental data as exact values. The focus is to identify an already localized nonlinearity with known structure. An optimization procedure is implemented using a metric involving the difference between the experimental kernel and the simulated orthogonal kernel from model updating, which is function of the unknown nonlinear parameters. In order to reduce the problems of convergence, the Kautz filter is used to decrease the number of samples to estimate. The proposed methodology is illustrated on a numerical example using a cantilever beam and its advantages and drawbacks are discussed in detail.

\section{Introduction}

Nowadays local methods for nonlinear finite element (FE) model updating based on vibration data are topics of active research motivated by practical engineering applications, mainly in the aerospace and automobile industries. $A$ representative example of a local nonlinear FE model updating is shown in [1] where the authors proposed a procedure based on the harmonic balance method to describe the dynamic behavior of structures with nonlinear joints. They illustrated their approach with an application to a nonlinear joint identification of aero-engine casing structures.

A classical and historical time domain approach with efficient and reliable results is the restoring force surface (RFS) method, described in standard textbooks [2]. The authors in [3] illustrate the method using a beam structure with bilinear stiffness. However, since the modal mass matrix must be known in advance, this approach proves to be difficult to implement for complex structures.

A more efficient time domain methodology is based on the proper orthogonal decomposition (POD) of the time histories. The POD is also known as Karhunen-Loève decomposition or principal component analysis (PCA). These concepts are mainly used in structural dynamics for compressing data, for example in [4]. The authors in [5] used the POD of a set of displacement vectors to identify the nonlinear parameters of a system using a metric based on the differences between the experimental and simulated PODs. The POD is a suitable and promising indicator for nonlinear model updating. However, the decomposition is a linear tool and there are some difficulties that must be overcome in the future. A solution to overcome

\footnotetext{
* Corresponding author.

E-mail addresses: sam.silva13@gmail.com, sam_silva13@hotmail.com (S. da Silva), scott.cogan@univ-fcomte.fr (S. Cogan), emmanuel.foltete@univ-fcomte.fr (E. Foltête).
} 
this drawback is to use a clustering technique, for example fuzzy c-means clustering or Loyd algorithm, to separate the data into groups and then to apply the POD to each cluster so as to preserve the character of the nonlinear effects [6]. Silva et al. [7] proposed a comparative study between these different classical metrics for nonlinear model updating based on vibration test data. Four approaches in the frequency and time domain were implemented: harmonic balance method, constitutive relation error, restoring force surface and Karhunen-Loève decomposition. The performance of each metric function was presented based on the form of the objective function and calculation time. The authors also discussed the applicability, limitations and differences between the investigated approaches with suggestions for improving each method.

In summary, the domain of nonlinear structural dynamics is not nearly as mature as its linear counterpart, and this is especially true for model updating techniques [8]. The principal reason for this limitation is due to the fact that the common metrics used in linear model updating are functions of relationships based on a unidimensional convolution or linear procedures, while nonlinear systems follow multidimensional convolutions. In this sense, the Volterra models are natural candidates to define metrics for updating. Classically speaking, the Volterra series is a technique suitable for describing higher-order frequency response functions (FRFs). However, the practical results produced have been limited by several factors, for instance:

(1) Numerical problems of convergence when a large number of samples is required to describe the Volterra kernels.

(2) The identification of high-order kernels, based on harmonic probing of the system equations of motion, is difficult to achieve for complex analytical models.

Fortunately, it is possible to express the Volterra series on an orthogonal basis, also known as a Wiener model, in order to minimize the above limitations [9] or [10]. In this sense, Laguerre and Kautz filters can be used to describe a discrete-time Wiener series thus allowing high-order kernels to be introduced in the model. However, in order to describe vibrating systems, the Kautz filter is better since it contains complex poles to represent the second-order dynamics [11,12] or [13]. Thus, the aim of this paper is to present an approach for updating nonlinear parameters using a local metric involving a Wiener series with an orthogonal basis based on a Kautz filter. The results are illustrated with a numerical simulation using a nonlinear cantilever beam with a local nonlinearity. The organization of the paper is as follows. In the next section, the discrete-time Wiener series and Kautz filter is briefly reviewed. Section 3 describes the proposed metrics for nonlinear model updating using the Wiener series. The numerical application is discussed in Section 4. Finally, the final remarks are summarized in Section 5 .

\section{Discrete-time Wiener series with a Kautz filter}

The Volterra models relate the output $x(k)$ of a dynamic system to its input $u(k)$ through $\eta$ th-order Volterra kernels $h_{\eta}\left(n_{1}, \ldots, n_{\eta}\right),[9,10]$. The main drawback of Volterra models is over-parametrization. Fortunately, it is possible to describe the Volterra kernels using an orthonormal basis $\psi_{i_{j}}\left(n_{j}\right)$, for example, Laguerre or Kautz functions. The Wiener series is an orthogonal expansion of the classical Volterra series. In practice, the Volterra kernels are approximated by a finite number $M$ of filters

$$
h_{\eta}\left(n_{1}, n_{2}, \ldots, n_{\eta}\right) \approx \sum_{i_{1}=1}^{M} \cdots \sum_{i_{\eta}=1}^{M} \alpha\left(i_{1}, \ldots, i_{\eta}\right) \prod_{j=1}^{\eta} \psi_{i_{j}}\left(n_{j}\right),
$$

where $M$ is the number of orthogonal filters used to approximate the kernels and the coefficients $\alpha\left(i_{1}, \ldots, i_{\eta}\right)$ are projections of the kernels onto an orthogonal basis $\psi_{i_{j}}\left(n_{j}\right)$. It is assumed that the system is causal and stable.

By ignoring the dynamics from higher-order kernels, the discrete-time Wiener series is represented by the following expression [10]:

$$
x(k)=\sum_{\eta=1}^{N} \sum_{i_{1}=1}^{M} \cdots \sum_{i_{\eta}=1}^{M} \alpha\left(i_{1}, \ldots, i_{\eta}\right) \prod_{j=1}^{\eta} l_{i_{j}}(k),
$$

where $N$ is the order and $l_{i_{j}}(k)$ is the input signal (excitation force) $u(k)$ filtered by the orthogonal basis $\psi_{i_{j}}\left(n_{j}\right)$ :

$$
l_{i_{j}}(k)=\sum_{\tau_{j}=0}^{\varepsilon} \psi_{i_{j}}\left(\tau_{j}\right) u\left(k-\tau_{j}\right),
$$

where $\varepsilon$ is the quantity of lag memory. In the present paper only the two first kernels for the Volterra series are used to describe the input-output data. Taking into account the symmetry property of kernels yields

$$
\begin{aligned}
& h_{1}\left(n_{1}\right)=\sum_{i_{1}=1}^{M_{1}} \alpha\left(i_{1}\right) \psi_{i_{1}}\left(n_{1}\right), \\
& h_{2}\left(n_{1}, n_{2}\right)=\sum_{i_{1}=1}^{M_{2}} \sum_{i_{2}=1}^{i_{1}} \alpha\left(i_{1}, i_{2}\right) \psi_{i_{1}}\left(n_{1}\right) \psi_{i_{2}}\left(n_{2}\right),
\end{aligned}
$$


where $M_{1}$ and $M_{2}$ are the number of orthogonal filters to identify the first $\alpha\left(i_{1}\right)$ and second $\alpha\left(i_{1}, i_{2}\right)$ orthogonal kernels, respectively.

Several types of orthogonal functions $\psi_{i_{j}}\left(n_{j}\right)$ can be used to represent the kernels $h_{1}\left(n_{1}\right)$ and $h_{2}\left(n_{1}, n_{2}\right)$ in Eqs. (4) and (5), for example, the polynomials of Chebyshev or Legendre. However, these functions are not directly related to the dynamic system by difference equations. Thus, the number of terms in Eqs. (4) and (5) must be high.

Fortunately, the Kautz filters are very effective in representing the orthogonal kernels. The Kautz filters contain poles with information on the dominant dynamics of the system [11-13]. Moreover, the poles in a Kautz filter are able to drastically decrease the number of parameters required to estimate the kernels $\alpha\left(i_{1}\right)$ and $\alpha\left(i_{1}, i_{2}\right)$. The Kautz filter $\Psi_{n}(z)$ has a pair of complex poles given by $\beta=\sigma+j \omega$ and $\bar{\beta}=\sigma-j \omega$. These poles are thus defined by two real scalars $b$ and $c$, where $|b|<1$ and $|c|<1$ for stable poles. This filter provides a good representation of the second-order dynamics of a vibrating system.

An important and difficult task to overcome is the definition of the poles $\beta$ and $\bar{\beta}$ used to build the filters. A procedure for estimating the poles and the kernels $\alpha\left(i_{1}\right)$ and $\alpha\left(i_{1}, i_{2}\right)$ simultaneously in an iterative way was described in [14]. Another approach was proposed in [15] considering generalized orthonormal bases. Meanwhile, in structural dynamics the linear mass $\mathbf{M}$, damping $\mathbf{C}$, and stiffness $\mathbf{K}$ matrices are often available from the finite elements method (FEM) or other previous analysis. So, this information can be used to estimate the Kautz poles in the $z$ plan. The dominant complex pole of the linear model can be chosen as the Kautz pole. Rosa et al. [14] concluded that the linear dominant complex pole of the system could be effectively used as Kautz parameter. However, the choice of the Kautz poles based on the linear portion of the system dynamics should only be done if the nonlinear system is very simple. In the present paper, the poles are defined using a trial-and-error procedure based on the response frequency of the system.

The elements in a set of Kautz filters are given by [12-14]:

$$
\begin{aligned}
& \Psi_{2 n}(z)=\frac{\sqrt{\left(1-c^{2}\right)\left(1-b^{2}\right) z}}{z^{2}+b(c-1) z-c}\left[\frac{-c z^{2}+b(c-1) z+1}{z^{2}+b(c-1) z-c}\right]^{n-1}, \\
& \Psi_{2 n-1}(z)=\frac{\sqrt{1-c^{2}} z(z-b)}{z^{2}+b(c-1) z-c}\left[\frac{-c z^{2}+b(c-1) z+1}{z^{2}+b(c-1) z-c}\right]^{n-1},
\end{aligned}
$$

where the constants $b$ and $c$ are relative to the poles $\beta$ and $\bar{\beta}$ through the relations

$$
\begin{aligned}
& b=\frac{(\beta+\bar{\beta})}{(1+\beta \bar{\beta})}, \\
& c=-\beta \bar{\beta} .
\end{aligned}
$$

Eqs. (6) and (7) are used for filtering the signals of input $u(t)$, and, then they are substituted in Eq. (3) to obtain the signals $l_{i_{1}}(k)$ and $l_{i_{2}}(k)$. The impulse response functions (IRF) of Eqs. (6) and (7) are recorded to estimate the physical kernels.

\section{Metrics for nonlinear model updating using Wiener series and Kautz filter}

The use of the first kernel, the classical impulse response function, or in the frequency domain the frequency response function, is commonly employed in the linear model updating techniques [16]. However, applications in the literature of the high-order Volterra kernels are relatively uncommon, for example, using $h_{2}\left(n_{1}, n_{2}\right)$ or $h_{3}\left(n_{1}, n_{2}, n_{3}\right)$ to construct an objective function for a local nonlinear updating of a large FE model.

The major advantage in the Volterra series can be seen in Eqs. (4) and (5): the separation between two contributions, one linear, $h_{1}\left(n_{1}\right)$, and another nonlinear, $h_{2}\left(n_{1}, n_{2}\right)$. The idea in the present paper is to compare the second-order orthogonal Wiener kernel extracted from the experimental data, designated here by $\alpha\left(i_{1}, i_{2}\right)_{\text {exp }}$, with another simulated Wiener kernel obtained from a mathematical model of the system for updating, for example a FE model of the structure. This new kernel is designated $\alpha\left(i_{1}, i_{2}\right)_{\text {mod }}$. One could also use the physical kernel $h_{2}\left(n_{1}, n_{2}\right)_{\text {exp }}$ and $h_{2}\left(n_{1}, n_{2}\right)_{\text {mod }}$. However, the sizes of the matrices $h_{2}\left(n_{1}, n_{2}\right)_{\text {exp }}$ and $h_{2}\left(n_{1}, n_{2}\right)_{\text {mod }}$ are bigger than the orthogonal kernels $\alpha\left(i_{1}, i_{2}\right)_{\text {exp }}$ and $\alpha\left(i_{1}, i_{2}\right)_{\bmod }$. The problem here is to choose a metric capable of smoothly representing the differences between the two matrices $\alpha\left(i_{1}, i_{2}\right)_{\exp }$ and $\alpha\left(i_{1}, i_{2}\right)_{\text {mod }}$. This metric is similar to the objective function involving the proper orthogonal modes (POD) [5]. Hence, the same drawbacks observed with the POD also appear in the proposed method. It is also necessary to integrate the nonlinear equations of motion at each model updating iteration. Moreover, the problem of identifying the Wiener kernels is more complex than the extraction of the POD. The difficulties aside, the Wiener kernels have the potential of treating a nonlinear updating problem independently from the linear updating problem.

Two objective functions involving the Wiener kernels are proposed in this paper. The first one investigates the modal assurance criteria (MAC) between the experimental and model kernels. This function is given by

$$
\min _{\mathbf{p}} J(\mathbf{p})=-\frac{1}{M_{2}} * \sum_{j=1}^{M 2} \operatorname{MAC}\left(\alpha\left(i_{1}, i_{2}\right)_{\text {exp }}, \alpha\left(i_{1}, i_{2}\right)_{\text {mod }}\right),
$$

where the kernel $\alpha\left(i_{1}, i_{2}\right)_{\text {mod }}$ changes following the modification of the nonlinear parameters vector $\mathbf{p}$ in each iteration step. 
The second one is a metric employing the normalized quadratic error between the experimental and model kernels

$$
\min _{\mathbf{p}} J(\mathbf{p})=\frac{\left\|\alpha_{\text {exp }}-\alpha_{\text {mod }}\right\|^{2}}{\left\|\alpha_{\text {exp }}\right\|^{2}},
$$

where $\alpha_{\text {exp }}=\sum_{i_{1}=1}^{M_{2}} \sum_{i_{2}=1}^{M_{2}} \alpha\left(i_{1}, i_{2}\right)_{\text {exp }}$ and $\alpha_{\text {mod }}=\sum_{i_{1}=1}^{M_{2}} \sum_{i_{2}=1}^{M_{2}} \alpha\left(i_{1}, i_{2}\right)_{\bmod }$.

The next section presents a numerical test in order to illustrate this methodology for updating the local nonlinearities in mechanical structures.

\section{Numerical application}

To illustrate the proposed method, an aluminum cantilever beam with a local nonlinearity, shown in Fig. 1, is considered. The dimensions of the beam are $(0.5 \times 0.025 \times 0.005) \mathrm{m}$ of length, width and thickness, respectively. The nonlinear beam is modeled with six finite elements. Each node contains 2-degrees of freedom (dof): one for vertical deflection and one for the rotation. Thus, the whole structure has 12-dof. The nonlinearity is considered as a spring that exhibits a cubic stiffness. The mathematical model of the structure is described by

$$
\mathbf{M} \ddot{\mathbf{x}}+\mathbf{C} \dot{\mathbf{x}}+\mathbf{K x}+\mathbf{f}_{n l}\left(x_{11}, k_{n l}\right)=\mathbf{u},
$$

where the linear part, $\mathbf{M}, \mathbf{C}$ and $\mathbf{K}$, is assumed to be known, $\mathbf{x}=\left[\begin{array}{llll}x_{1} & x_{2} & \cdots & x_{12}\end{array}\right]$, the damping is simulated as proportional $\mathbf{C}=3 \times 10^{-5} \mathbf{K}$, $\mathbf{u}$ is the excitation force applied at the free end of the beam (dof 11$)$ and $\mathbf{f}_{n l}\left(x_{11}, k_{n l}\right)$ is the nonlinear force vector simulated by

$$
\mathbf{f}_{n l}\left(x_{11}, k_{n l}\right)=k_{n l} x_{11}^{3} \text {, }
$$

where $k_{n l}$ is the nonlinear cubic stiffness parameter to be identified. This local nonlinearity is given here by $k_{n l}=$ $1 \times 10^{8} \mathrm{~N} / \mathrm{m}^{3}$ and its location and structure are assumed to be known in advance.

Eq. (12) is solved by the Newmark and Newton-Raphson method to simulate the experimental responses. The sampling frequency is set to $F_{s}=2000 \mathrm{~Hz}$ with 2048 time samples. Fig. 2 shows the FRF plots (receptance) of the relationship between the displacement $x_{11}$ and the excitation in dof 11. Three different conditions are simulated: (1) linear responses with $k_{n l}=0,(2)$ nonlinear responses due to an input chirp signal having an amplitude of $0.05 \mathrm{~N}$ and a frequency range from

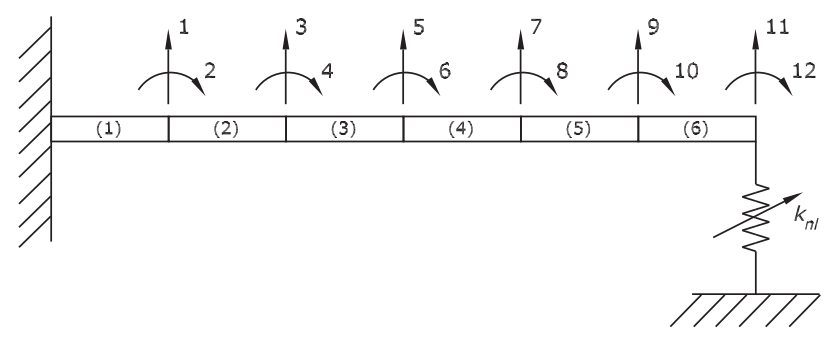

Fig. 1. Cantilever beam with a local nonlinearity.

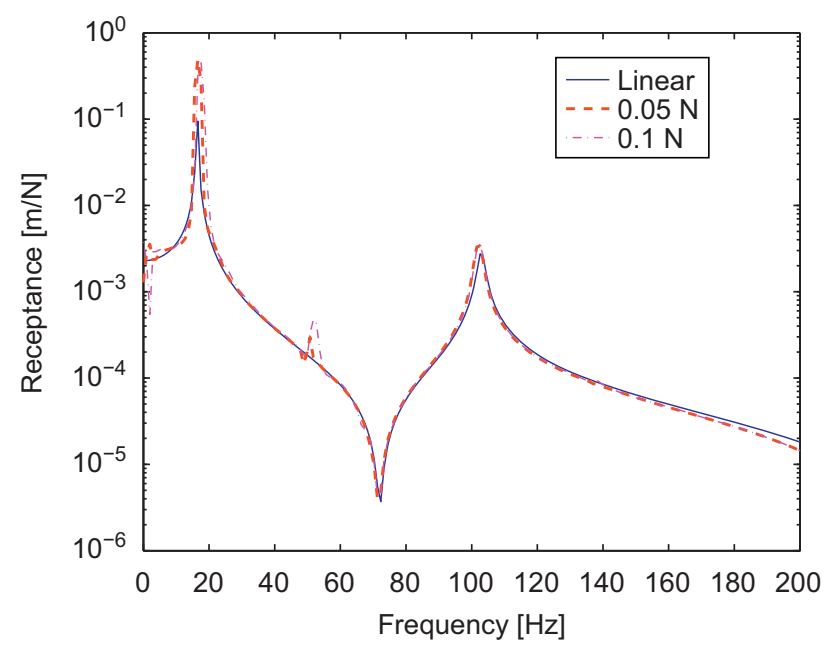

Fig. 2. Linear and non-linear frequency response function of the cantilever beam for several levels of chirp amplitude excitation. 
1 to $500 \mathrm{~Hz}$ in $1 \mathrm{~s}$, and (3) nonlinear responses due to an input chirp signal having an amplitude of $0.01 \mathrm{~N}$ and a frequency range from 1 to $500 \mathrm{~Hz}$ in $1 \mathrm{~s}$. The resonance peaks change when the amplitude is increased and one can see the presence of harmonics in the natural frequency close to $50 \mathrm{~Hz}$. This is a qualitative index to the presence of nonlinearity that affects primarily the first mode of the equivalent linear model. In this plot, the FRFs were estimated using the power spectral density and $H_{1}$ estimator [17].

The input-output signals for an excitation amplitude of $0.1 \mathrm{~N}$ were employed to estimate the experimental Wiener kernels and to verify the nonlinear identification procedure. In order to avoid numerical convergence and ill-posed problems the signals were normalized to a range of $[-1+1]$. The objective function proposed in Eqs. (10) and (11) involve only the second-order Wiener kernel. Once the linear parameters are assumed to be known, the pair of complex poles in first mode in the Laplace $s$ domain can be well estimated by $s_{1,2}=-\xi \omega_{n} \pm j \omega_{n} \sqrt{1-\xi^{2}}$. Since the objective function depends only on the second Wiener kernel and only this parameter is estimated and a frequency of $50 \mathrm{~Hz}$ (peak in Fig. 2) and $\xi=5 \times 10^{-2}$ is used. For this case, the continuous pole was computed by $s_{1,2}=-15.9 \pm 3.18 \times 10^{2} j$. This value is chosen with a procedure based on a qualitative analysis of Fig. 2 to find a candidate harmonic for the first mode. Further study is required to utilize more sophisticated and flexible methods to set up the Kautz filter discussed earlier. The goal here is only to illustrate the behavior and the shape of specific objective functions for model updating defined by Eqs. (10) and (11).

Meanwhile, since the Kautz filter is a discrete-time system, the equivalent pole in the $z$ domain is obtained from the relation $\beta=e^{s T}$, where $T=1 / F_{s}$. The discrete-time poles are then given by $\beta=0.98 \pm 0.16 j$. If the Kautz pole is well defined one can use a low number of $M$ of Kautz filters. In the present example, $M=2$ is assumed to be a good number of Kautz filters once the Kautz pole has been estimated by graphical analysis as in Fig. 2. The quantity of lag memory used was set by $\varepsilon=600$. This value is chosen because it was observed that for values bigger than this lag $\varepsilon$, the response had low energy levels and the signal-noise ratio was poor. Finally, the identified experimental orthogonal kernel $\alpha\left(i_{1}, i_{2}\right)_{\exp }$ is given by

$$
\alpha\left(i_{1}, i_{2}\right)_{\exp }=\left[\begin{array}{cc}
0.035 & -0.098 \\
-0.098 & 0.066
\end{array}\right] .
$$

After obtaining the experimental orthogonal kernel $\alpha\left(i_{1}, i_{2}\right)_{\text {exp }}$, the physical kernel $h_{2}\left(n_{1}, n_{2}\right)$ can be found by projection in Eq. (5) once the impulse response of the Kautz filter is known. The graphical result of the second kernel identification is presented in Fig. 3.

The shape of the objective function based on the proposed time domain metrics for nonlinear systems using the Wiener series with Kautz filter will now be presented. Parameter variations over a wide range are used to assess the objective function. Indeed, the search limits were defined from $1 \times 10^{5}$ to $1 \times 10^{10} \mathrm{~N} / \mathrm{m}^{3}$. Fig. 4 shows the objective function based on Eq. (10). The exact value of the nonlinear parameter $k_{n l}$ is indicated in this plot as a reference. Fig. 5 shows the residual from Eq. (11) for the same amplitude. The cost functions are not flat and there is a large gradient near to the minimum value in the range of the search based on Eq. (10). The first remark is that the curves tend to be convex for this amplitude level and hence well adapted to conventional local optimization procedures. In all cases the minimums of the curves correspond to the exact value of the nonlinear parameter $k_{n l}$. However, over the range studied local minimum can occur.

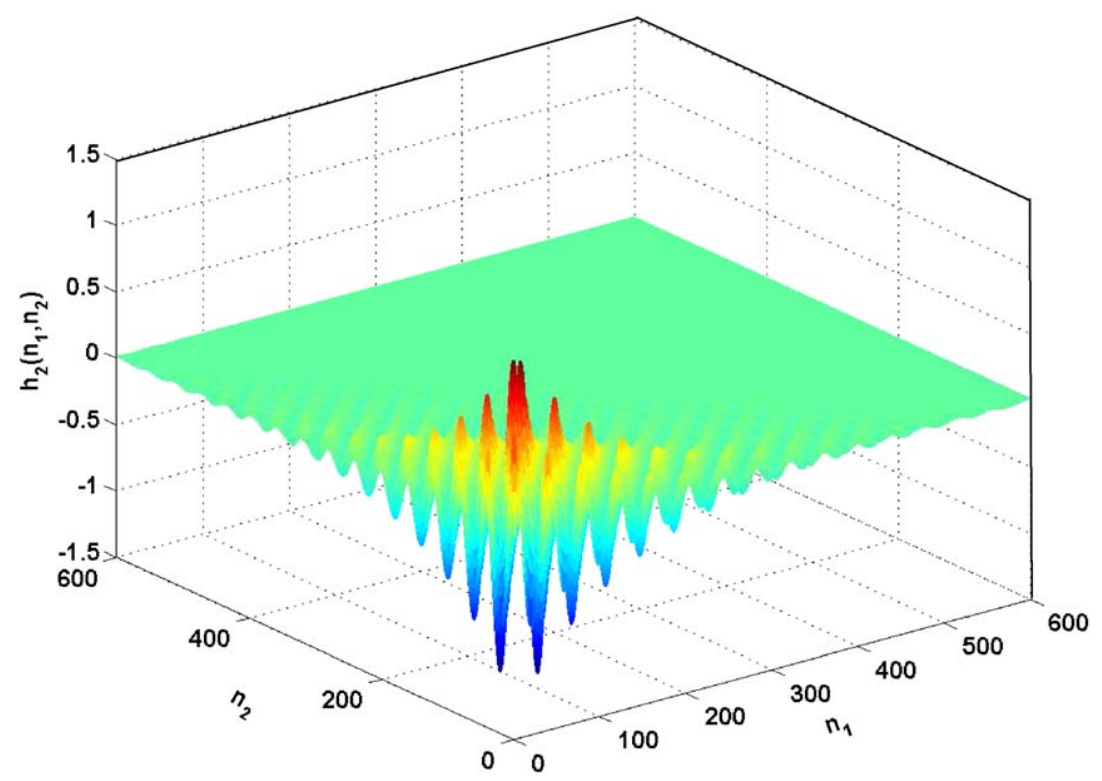

Fig. 3. Experimental second-order kernel $h_{2}\left(n_{1}, n_{2}\right)$ from a Kautz-Wiener model for an amplitude of $0.1 \mathrm{~N}$. 


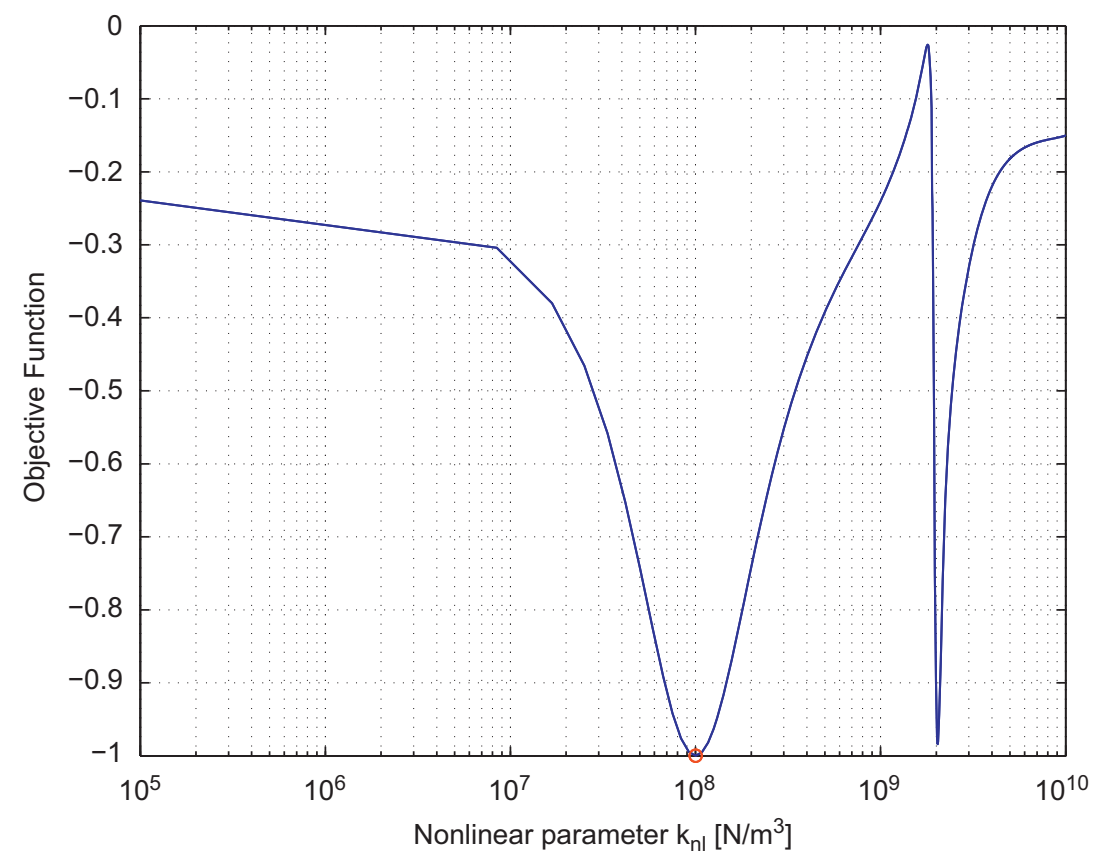

Fig. 4. Evolution of the residuals given by Eq. (10) as a function of the non-linear updating parameter with the exact value indicated by 0 .

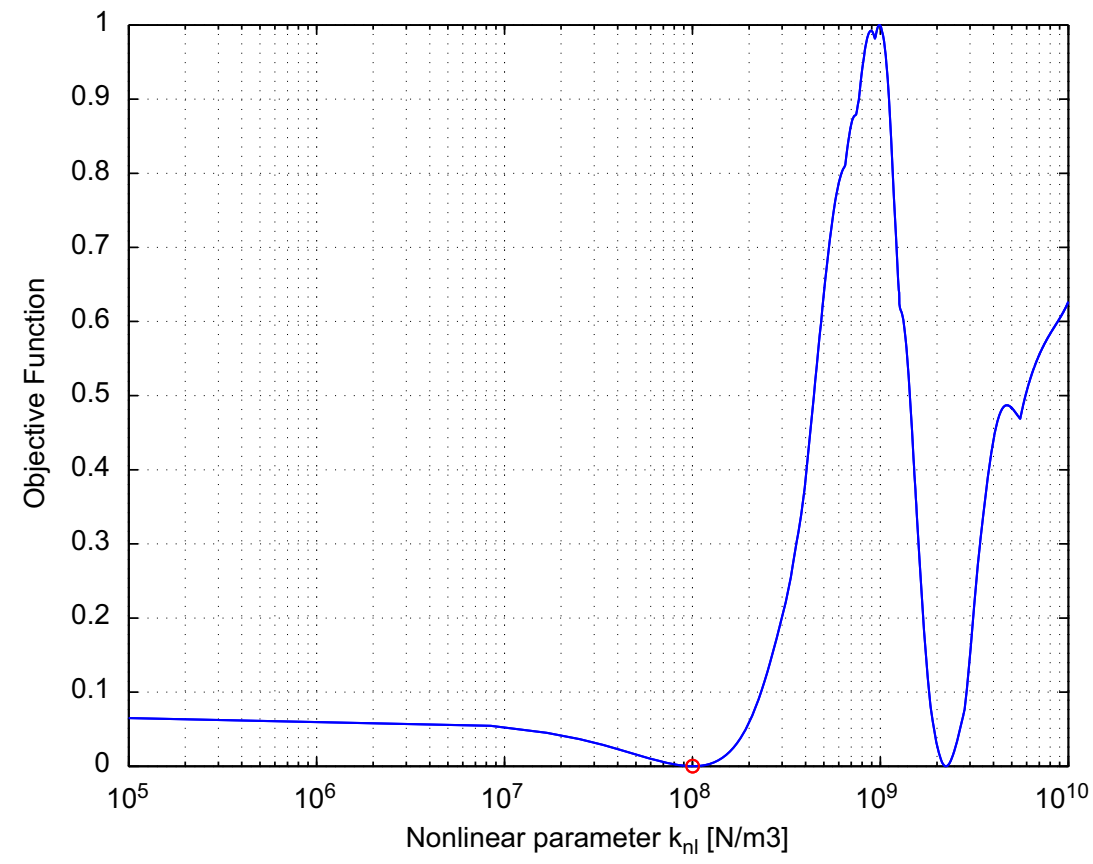

Fig. 5. Evolution of the residuals given by Eq. (11) as a function of the nonlinear updating parameter with the exact value indicated by 0 .

The curves can become multimodal and other very flat, which can be more difficult to handle in an optimization loop. The objective function based on Eq. (11) also has a wide range where the values are almost constant and conventional optimization procedures would be inefficient in this case. In the tests, one also note that this technique is very sensitive to the Kautz pole used as well as the number of filters. When these values are not correctly defined, the kernel was poorly estimated and the results can diverge from the real parameters because the shape of the objective function can change considerably. The same remark holds for large variations in excitation amplitude due to its influence on the level of nonlinearity. 


\section{Final remarks}

The approach described in this paper employs the second-order Wiener kernel using the Kautz filter as a metric for nonlinear model updating. The methodology was illustrated on an academic model and it was shown that the proposed metrics have practical applicability to improve the updating of the local nonlinear parameters in mechanical systems when the localized nonlinearity has a well known structure. The technique can also be effectively used to separate the nonlinear and linear parts of time responses. This property is very promising in order to simplify the model updating of industrial structures where the behavior is nonlinear even at low levels of excitation. It is also worthwhile to note that the chosen Kautz pole is directly related to the localized nonlinear parameter. In applications where the nonlinear behavior is strong it may be necessary to include another higher-order kernel, for example, $\alpha\left(i_{1}, i_{2}, i_{3}\right)$, or even to propose an hybrid metric. Future study is required to provide a way to set the Kautz filter for the system, mainly when the application is a complex or industrial structure. A procedure to improve the shape of the objective function in order to reduce the multimodal behavior is also the subject of further work.

\section{Acknowledgment}

The authors would like to thank the CNES-Toulouse for their generous financial support. The first author also acknowledges the Research Foundation of Ilha Solteira (FEPISA, Brazil).

\section{References}

[1] M. Böswald, M. Link, Identification of non-linear joint parameters by using frequency response residuals, in: International Conference on Noise and Vibration Engineering, 2004, pp. 3121-3140.

[2] K. Worden, G.R. Tomlinson, Nonlinearity in Structural Dynamics, Institute of Physics Publishing, London, 2001

[3] G. Kerschen, J.C. Golinval, K. Worden, Theoretical and experimental identification of a non-linear beam, Journal of Sound and Vibration 244 (4) (2001) 597-613.

[4] S. Silva, M. Dias Junior, V. Lopes Junior, M.J. Brennan, Structural damage detection by fuzzy clustering, Mechanical Systems and Signal Processing 22 (2008) 1636-1649.

[5] V. Lenaerts, G. Kerschen, J.C. Golinval, Proper orthogonal decomposition for model updating of non-linear mechanical system, Mechanical Systems and Signal Processing 15 (1) (2001) 31-43.

[6] A.M. Yan, G. Kerschen, P. de Boe, J.C. Golinval, Structural damage diagnosis under varying environmental conditions-part ii: local PCA for non-linear cases, Mechanical Systems and Signal Processing 19 (4) (2005) 865-880.

[7] S. Silva, S. Cogan, E. Foltête, F. Buffe, Metrics for non-linear model updating in mechanical systems, Journal of the Brazilian Society of Mechanical Sciences and Engineering 31 (1) (2009) 27-34.

[8] G. Kerschen, K. Worden, A.F. Vakakis, J.C. Golinval, Past, present and future of nonlinear system identification in structural dynamics, Mechanical Systems and Signal Processing 20 (2006) 502-592.

[9] M. Schetzen, The Volterra and Wiener Theories of Nonlinear Systems, Wiley, New York, 1980

[10] W.J. Rugh, Nonlinear System Theory-The Volterra/Wiener Approach, Johns Hopkins University Press, Baltimore, MD, 1991.

[11] S. Silva, M. Dias Jr., V. Lopes Jr., Identification of mechanical systems through Kautz filter, Journal of Vibration and Control 15 (2009) 849-865.

[12] P.M. Vand den Hof, P.S.C. Heuberger, J. Bokors, System identification with generalized orthonormal basis function, Automatica 31 (12) (1995) 1821-1834.

[13] B. Wahlberg, System identification using Kautz models, IEEE Transactions on Automatic Control AC-39 (1994) 1276-1282.

[14] A. Rosa, R.J.G.B. Campello, W.C. Amaral, Choice of free parameters in expansions of discrete-time Volterra models using Kautz functions, Automatica 43 (2007) 1084-1091.

[15] A.Y. Kibangou, G. Favier, M. Hassani, Selection of generalized orthonormal bases for second-order Volterra filters, Signal Processing 85 (12) (2005) $2371-2385$.

[16] J.A. Pereira, W. Heylen, P. Sas, Model updating and failure detection based on experimental FRFS: case study in space frame structure, in: 19th International Conference on Noise and Vibration Engineering (ISMA), 1994, pp. 669-681.

[17] P. Stoica, R.L. Moses, Introduction to Spectral Analysis, Prentice-Hall, Englewood Cliffs, NJ, 1997. 